\title{
Моделирование пространственно-временной динамики двухволнового кольцевого волоконного нелинейного микрорезонатора
}

\author{
В.А. Разуков ${ }^{*}$, Л.А. Мельников \\ Саратовский государственный технический университет имени Ю.А. Гагарина \\ *E-mail: razukov.vad@gmail.com
}

DOI: 10.31868/RFL2020.166-167

Очевидно, что возможность предсказать поведение электромагнитного поля в микрорезонаторах имеет огромное практическое значение. Так как режим работы таких резонаторов сильно нелинейный, то исследование их динамики возможно лишь с применением методов численного моделирования, причем с привлечением моделей, адекватных в описании происходящих процессов, но и не требующих длительных расчетов. Основным [1] является модовый подход, при котором поле внутри микрорезонатора разбивается по продольным модам, и записываются уравнения для комплексных амплитуд этих мод, зависящих от времени. Результатом будет система десятков или даже сотен обычных граничных нелинейных уравнений, решение которых на компьютере является нетривиальной задачей. Например, из-за нелинейности, в уравнениях появляются суммы всех возможных произведений мод, и расчеты потребуют значительных временных и машинных ресурсов. Кроме того, чтобы рассчитать профиль поля во времени, требуется сложить поля мод, что тоже занимает много времени, если таких мод очень много. Это все примеры методов разложения в спектр. Альтернативным подходом к задаче динамики поля в микрорезонаторе является разностная схема, основанная на уравнениях переноса. [2], успешно примененная для моделирования рамановских и ВРМБ лазеров. Данная работа посвящена дальнейшему улучшению численной модели [2], и анализу результатов, полученных с помощью данной методики.

Уравнения, описывающие распространение импульса в внутри микрорезонатора, задаются следующим образом:

$$
\begin{aligned}
& 2 i\left(\frac{\partial F}{\partial t}+v \frac{\partial F}{\partial z}\right)+D \frac{\partial^{2} F}{\partial z^{2}}+2 \chi\left(|F|^{2}+2|B|^{2}\right) F=0, \\
& 2 i\left(\frac{\partial B}{\partial t}-v \frac{\partial B}{\partial z}\right)+D \frac{\partial^{2} B}{\partial z^{2}}+2 \chi\left(2|F|^{2}+|B|^{2}\right) B=0 .
\end{aligned}
$$

Граничные условия имеют вид: $F(0)=\sqrt{1-R} \sqrt{1-r} F(L)+\sqrt{R} \sqrt{A} \sqrt{1-r}+$ $\sqrt{r} B(0) ; B(L)=\sqrt{1-R} \sqrt{1-r} B(0)-\sqrt{r}(1-r) F(L)+\sqrt{R r} \sqrt{1-R} \sqrt{A}$.

Здесь $F$ и $B$ - поля волн, распространяющихся по и против часовой стрелок, $D<0$ - коэффициент ДГС, $v$ - групповая скорость, $\chi$ - коэффициент фазовой кросс и самомодуляции, $R$ - коэффициент отражения ответвителя, $r$ - коэффициент отражения зеркала внутри резонатора, $A-$ интенсивность внешней накачки, $L-$ длина резонатора.

Мы учитываем дисперсию и нелинейность микрорезонатора, ответвитель, и зеркало, расположенное в случайном месте волокна. Кроме упомянутых эффектов, так же может присутствовать модуляционная неустойчивость. Для решения задачи применяется эффективная разностная схема второго порядка «Кабаре» [3]. На рисунке приведен пример учета невзаимного сдвига по фазе в 
волокне, вызванного, например, вращением, и рэлеевского рассеяния на неоднородностях в среде резонатора [4].
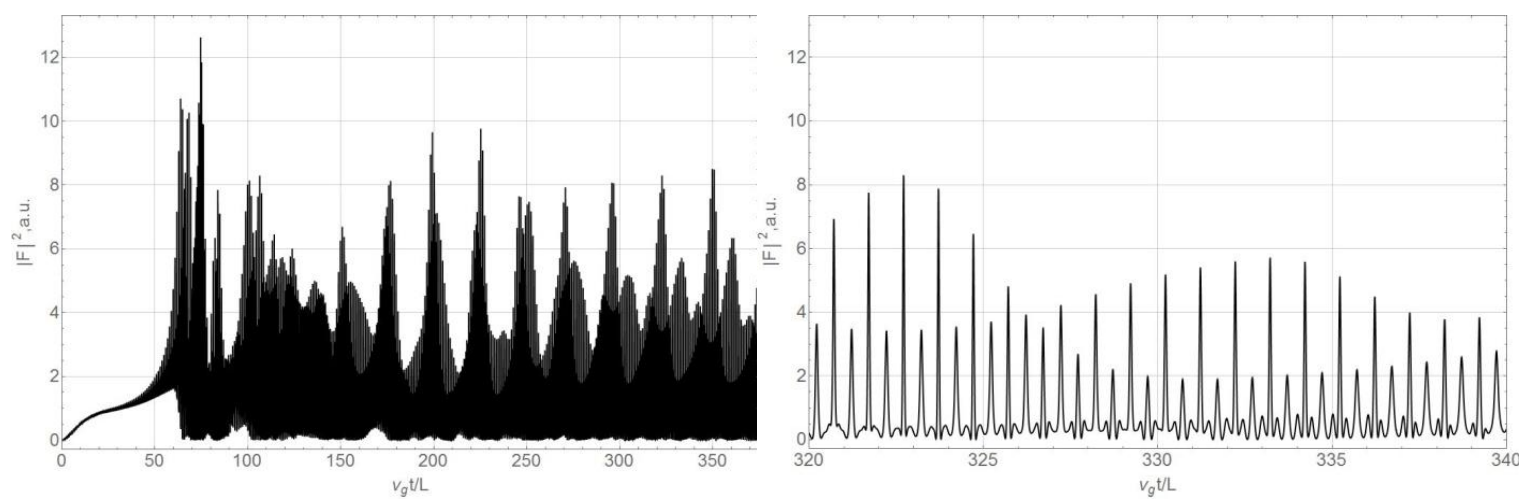

Рис. 1. Формирование оптической гребенки. Заметен сильный фоновый фазовый шум. Коэффициент модуляции $\chi=0,25$. Коэффициент линейной связи волн $r=$ 0.001. Коэффициент потерь 0.0001 .

Таким образом, использование явно-неявной схемы «Кабаре» позволяет с легкостью учитывать необходимые нелинейные эффекты в наблюдаемой системе. Это практически невозможно с модовым подходом, так как количество уравнений увеличивается в несколько раз при вводе каждого нового эффекта, с соответствующим ростом необходимого расчетного времени.

\section{Литература}

[1] T. Herr, V. Brasch et al, Nature Photonics, 8, 145-152 (2014).

[2] V.A. Razukov and L. A. Melnikov, in Saratov Fall Meeting 2015: Third International Symposium on Optics and Biophotonics and Seventh Finnish-Russian Photonics and Laser Symposium (PALS), 9917, 788 - 792 (2016)

[3] В.М. Головизнин, А.А. Самарский, Математическое моделирование. 10, 86-100 (1998).

[4] Разуков В.А., Мельников Л.А., Известия Саратовского университета. Новая серия. Серия Физика. 20(1). 64-71 (2020). 